\title{
Propuesta de un marco educativo para una sociedad sostenible
}

Recibido: 21 de marzo de 2018 / Revisado: 10 de abril de 2018

Aceptado: 30 de abril de 2018 / Publicado: 12 de julio de 2018

SEGURA CHECA, JUAN ANTONIO

Departamento de Biología Molecular y Bioquímica

Universidad Pública de Málaga, España

jsegura@uma.es

DOI: 10.24310/IJNE1.1.2018.4946

\section{RESUMEN}

La sociedad actual hace tiempo que sigue una trayectoria incompatible con el sostenimiento de los ecosistemas naturales de la tierra. Este comportamiento está caracterizado por una visión antropocéntrica de nuestra relación con la naturaleza, implícito en la definición tradicional del concepto de sostenibilidad, y por un paradigma social consumista que ahonda en los comportamientos insostenibles. Para tomar la senda hacia la sostenibilidad sería necesario, pues, actuar sobre ambos rasgos de nuestra sociedad, adquiriendo una visión ecocéntrica de nuestro papel en la biosfera, y abordando el cambio del paradigma social consumista. En este trabajo se identifica al proceso educativo como el único capaz de impulsar tales cambios, formando individuos con las actitudes, aptitudes y los valores precisos para que puedan hacer suyos los problemas sociales y medioambientales existentes, y acometer las transformaciones necesarias. Para ello, se propone un marco educativo, constituido por axiomas que se implementan bajo un paraguas epistemológico y ético, que promueva el diseño de las acciones educativas necesarias. Los axiomas se han diseñado

\section{ABSTRACT}

Proposal of an educational framework for a sustainable society

Society has long been tracing a path that it is incompatible with the preservation of healthy natural ecosystems. This behavior is characterized by an anthropocentric view of our place in the biosphere, that it is implicit in the very definition of the sustainability concept. In addition, a consumerist social paradigm deepens in our unsustainable behavior. In order to take the sustainability path it would be necessary to act upon both attributes of our society, favoring an ecocentric view of our place in the biosphere and taking over the consumerist paradigm shift. In this article it is proposed that those changes are only possible through education, and its aim is to endorse people with the skills, attitudes and values necessary to identify the societal problems as self problems and undertake the necessary transformations. To achieve this, it is proposed an educational framework, constituted by axioms that are implemented under an epistemological and ethical umbrella, that promotes the design of 
con la intención de despertar la conciencia medioambiental de los estudiantes, su participación social, la cooperación entre géneros y el uso racional de la tecnología. La epistemología propuesta está basada en la lógica de funcionamiento de los sistemas complejos, lo cual facilitaría un aprendizaje profundo, frente al aprendizaje superficial actualmente mayoritario, y así interpretar correctamente las vivencias diseñadas mediante los axiomas educativos anteriores. Y finalmente, sería necesario la aceptación implícita de una ética humanitarista, no excluyente, que abarcara los valores intrínsecos asociados al medio natural. Este marco educativo facilitaría un aprendizaje profundo, transformador y compatible con una visión sostenible de nuestra sociedad.

PALABRAS CLAVE: Paradigmas, educación, sostenibilidad, ética, axiomas educativos, complejidad. the necessary educational actions. The axioms have been designed with the intention of awakening students' environmental awareness, their social participation, gender cooperation and the rational use of technology. In this process it is proposed the functional logic of complex systems as the basis of an epistemology that will contribute to knowledge acquisition. This will lead to a deep learning, in opposition to a surface learning characteristic of today's education, and to a correct interpretation of the designed educational experiences. Finally, it would be necessary to accept a non-discriminating humanitarianism ethic that will assign intrinsic values to the natural environment. This axiomatic, epistemological and ethical system will generate educational experiences leading to a transforming deep learning, and compatible with a sustainable view of society.

KEYWORDS: Paradigms, education, sustainability, ethics, axioms, complexity.

\section{Introducción}

\subsection{Sostenibilidad en el siglo XXI}

El concepto de sostenibilidad tiene una corta pero intensa vida. Nació en 1987, cuando la World Commission on Enviroment and Development, en el marco de las Naciones Unidas, definió el desarrollo sostenible como "aquel que satisface las necesidades del presente sin perjuicio de que futuras generaciones satisfagan las suyas propias" (Harlem, 1987) El nuevo concepto de sostenibilidad hizo posible la convivencia de dos corrientes contrapuestas hasta entonces: el crecimiento económico y la defensa del medio ambiente. Para ello, se utilizó el término de desarrollo sostenible como la meta a alcanzar, compatibilizando los objetivos del crecimiento económico con los límites medioambientales. Si el crecimiento económico podía hacerse sostenible, no había que preocuparse de sus efectos sobre el medio ambiente. De esta forma, los defensores del medio ambiente ya no eran estigmatizados como enemigos del progreso, y las corporaciones que sustentaban el crecimiento económico no amenazaban los ecosistemas; ambos tendrían el mismo objetivo, el desarrollo sostenible. Sin embargo, el concepto 
de sostenibilidad nació impregnado de una visión antropocéntrica, en la que se hablaba de nuestras necesidades, sin mencionar las necesidades del resto de la biosfera (Imran, Alam \& Beaumont, 2011). No obstante, esto se comprende al contextualizarlo históricamente, ya que el concepto de medio ambiente es sólo media generación más antiguo que el de sostenibilidad. Fue en 1970, cuando la Organización de Naciones Unidas inauguró el Día de la Tierra en el calendario, y el concepto de medio ambiente relevó al de conservacionismo como nuevo paradigma para interpretar nuestra relación con el entorno natural (Appleton, 2006) se dejó atrás entonces la visión de lo natural como algo a conservar en reductos protegidos y apartados del resto de la naturaleza sometida a explotación.

Esta nueva visión, o "paradigma de progreso", ha hecho posible que la sostenibilidad entre en el siglo XXI como garante de toda una nueva economía: la economía verde. El surgimiento de nuevas tecnologías, como la solar, la eólica o los biocombustibles, junto con una nueva forma de ver la ciencia y la ingeniería, ambas denominadas verdes, ha alimentado también la esperanza de alcanzar algún día este deseado desarrollo sostenible (Mulvihill, Beach,Zimmerman \& Anastas, 2011). Bajo este paraguas, la economía ha seguido creciendo, junto con el consumo, la acumulación de residuos y la pérdida de biodiversidad, llegando al límite de la capacidad de soporte de la Tierra (Daily \& Ehrlich, 1996). Mientras tanto, la sociedad trataba de sustanciar el concepto de desarrollo sostenible en políticas que, una tras otra, se disolvían en el paradigma del crecimiento económico, el cual era impulsado aún más por las nuevas economías de los países emergentes. Piénsese por ejemplo en el fracaso de poner límites a la emisión de gases de efecto invernadero en sucesivas cumbres sobre el cambio climático.

La sostenibilidad sigue siendo hoy día un concepto antropocéntrico, estando muy asociado a conceptos sociales. Un análisis de los trabajos publicados sobre sostenibilidad en Web of Science hasta 2010 (Burger et al., 2012), ha puesto de manifiesto que en el 48\% de estos trabajos, la sostenibilidad era estudiada en el ámbito del desarrollo o la economía, un 17\% en el ecológico y un 12\% en el energético. Sólo en un 2\% aparece asociado el término límites. Este sesgo en la contextualización del concepto de sostenibilidad lleva implícita la idea de Naturaleza como recurso, y además el que sólo nosotros, los humanos, poseemos valores intrínsecos y derechos morales (van Gorp, 2014). Esta visión contrasta con la que tienen ciertas culturas ancestrales que han vivido más en armonía con la naturaleza. Oren Lyons, nativo Onondaga, tribu Iroquesa declarada Patrimonio Nacional Inmaterial en los Estados Unidos, nos recuerda que "lo que tu gente llama recursos naturales, para nosotros son nuestros ancestros". La visión antropocéntrica de la sostenibilidad se preocupa, al fin y al cabo, de que nuestros descendientes vivan en condiciones comparables o mejores que las que disfrutamos hoy, sin poner en entredicho el que estas condiciones sean justas o sostenibles. Es este el caldo de cultivo en el que se han desarrollado las energías verdes, pero éstas difícilmente podrán sustituir completamente a las tradicionales fuentes de energía fósiles si no se produce una disminución del consumo energético (de Castro, Mediavilla, Miguel \& Frechoso, 2013). El debate debe de ser reorientado, pues, hacia una visión ecocéntrica, en la que 
tanto las ciencias sociales como las tecnológicas tienen mucho que aportar. El modelo antropocéntrico del desarrollo sostenible debe de ser cuestionado también éticamente, ya que, según el World Business Council for Sustainable Development, nos conduce al desastre (WBCSD, 2010).

La postura que actualmente defienden las personalidades y organizaciones más destacadas en la defensa de la sostenibilidad y del medio ambiente, ya no es optimista sobre la cuestión de si estamos a tiempo de lograr la sostenibilidad y evitar el desastre. La nueva cuestión es ¿cómo podemos adaptarnos a ese nuevo escenario que nos sobreviene? El Worldwatch Institute ha publicado en su informe sobre el estado del mundo de 2013 un libro titulado Is sustainability still possible? (Robinson, 2013). Es muy revelador que uno de sus tres apartados se titule "Abrir en caso de emergencia", y en él se incluyan, entre otros, artículos sobre la gobernanza en casos de crisis y emergencias, la respuesta frente a catástrofes e incluso si el fin justifica los medios en los movimientos activistas radicales.

Es en este escenario, donde las políticas nacionales se ponen en entredicho, tanto por la comunidad científica como por parte de la sociedad más crítica, pero se sigue persiguiendo el aumento de PIB como meta principal para alcanzar el bienestar de la sociedad (Victor, 2010), donde existen movimientos ciudadanos que piensan y promueven cambios verdaderos pero su voz no alcanza al ámbito político, es en el que hay que trabajar en pos de una transformación de la sociedad, transformación que la prepare para lo que se avecina y que promueva una visión ecocéntrica del mundo (Imran et al., 2011). En esta visión predominaría una ética natural en un mundo más equitativo, justo y complejo (Rogers et al., 2012). El trabajo ahora es, pues, educar a la sociedad en este nuevo paradigma, investigando en nuevos conceptos educativos que hagan emerger una visión ecocéntrica como eje de pensamiento, y que genere nuevos ciudadanos que pongan en entredicho las viejas políticas y asunciones que sustentan la actual sociedad.

\subsection{Aproximaciones previas a una educación para la sostenibilidad}

Los conceptos de sostenibilidad y desarrollo sostenible han tenido un grado de implementación notablemente condicionado por su visión antropocéntrica. Aunque, en muchos aspectos, quizás no ha sido este, su antropocentrismo, el principal problema. Por ejemplo, repasando los diferentes programas de enseñanza a nivel nacional, se puede comprobar la escasa presencia del concepto de sostenibilidad en el sistema educativo, y esto nos hace reflexionar sobre el escaso calado que dicho concepto ha tenido en la sociedad. Sin embargo, no nos debería sorprender, ya que, aun siendo conceptos importantes, ¿cómo se implementa la noción de sostenibilidad en un sistema educativo?, ¿esperamos que una clase política que persigue el crecimiento económico tradicional siembre las bases educativas que acaben con el crecimiento? Por otra parte, el cambio a una visión ecocéntrica de la biosfera, con su alto grado de carga ética, hace aun más compleja la transición. Los cambios en el sistema educativo que lleven a esta trans- 
formación son más necesarios que nunca, aunque también es cierto que estos cambios no se producirán mediante cambios legislativos a la manera tradicional.

Los paradigmas educativos adoptados por los diferentes sistemas educativos tienen una gran trascendencia en el aprendizaje, de ahí la importancia de reflexionar críticamente sobre las bases filosóficas de nuestros sistemas educativos (Georgescu, 2008). Si bien en ciencia los paradigmas son reemplazados por otros (Khun, 2012), en filosofía y ciencias sociales pueden coexistir diferentes paradigmas filosóficos (Dean \& Kuhn, 2007) (Klahr \& Nigam, 2004).

El pragmatismo y neopragmatismo están basados en el legado de Dewey, y nacen de la visión que Dewey tenía del estudiante como perseguidor activo de su propio propósito u objetivo. Dewey veía a la escuela como un lugar donde no sólo se adquieren conocimientos, sino que también se aprende a vivir (Dewey, 2013). En esta visión de la docencia es muy importante el concepto de práctica reflexiva, donde el foco de la reflexión se centra en hechos y acciones (Dewey, 1910). Está, de hecho, muy extendida la pedagogía del aprender haciendo, con prácticas pedagógicas derivadas como la docencia y aprendizaje interactivos, escuelas democráticas, participación y aprendizaje basado en problemas. Muy actuales son las nuevas concepciones de la docencia denominadas orientaciones postmodernas relativistas. Sus fundamentos radican en el constructivismo social, dominado por una visión de la perspectiva de género y la educación inclusiva, y el relativismo científico, donde tienen una influencia grande conceptos como el principio de incertidumbre de Heissenberg y la mecánica cuántica probabilística, y las teorías del caos y sistemas complejos. El componente multicultural de este tipo de educación se ha llevado bastantes críticas, y le ha sido achacado la pérdida de valores tradicionales (Wessendorf, 2010). Finalmente, se ha venido en llamar filosofía social y política a una corriente educativa, amparada por movimientos progresistas, que promueve un cambio del modelo docente, desde el centrado en el paradigma de la enseñanza al centrado en el paradigma del aprendizaje, con el aprendiz en el foco del proceso (Barr \& Tagg, 1995). El concepto de emancipación del educando ha trascendido el marco docente transmisivo y ha abrazado un compromiso social, surgiendo los conceptos de educación transformadora y el de pedagogía crítica que han abordado la emancipación del pobre y el oprimido. Clave en la interpretación de los conceptos de la filosofía social ha sido la aportación de Freire (Freire, 2005). Esta filosofía educativa ha estado más comprometida con los conceptos de sostenibilidad y desarrollo sostenible, aunque su implementación, al igual que en el resto de paradigmas educativos, ha conllevado la transmisión y perpetuación de la mentalidad consumista occidental, plasmada en el paradigma social predominante que lo impregna todo.

Las Naciones Unidas, en el marco del programa Educación Para Todos, ha estimulado, desde principios del siglo XXI, a tomar la educación como el cimiento del desarrollo sostenible. En este sentido la UNESCO proclamó el decenio 2005/2014 como la década de la educación para el desarrollo sostenible, con el fin de integrar el desarrollo sostenible dentro de los sistemas educativos a todos los niveles, y 
conseguir que la educación pueda ser un agente para el cambio. A pesar del gran interés mostrado por múltiples organizaciones educativas, su implementación no ha logrado cambiar los paradigmas educativos tradicionales, ya que se sólo se han logrado implementar acciones puntuales como cursos y proyectos para el desarrollo sostenible. En su informe, la UNESCO reconoce que la consecución del objetivo de este programa no se puede llevar a cabo mediante asignaturas independientes, sino transformando la propia filosofía educativa subyacente. Los cambios necesarios deberían abordarse desde perspectivas socioculturales, ambientales y económicas, abarcando desde los derechos humanos, la paz, igualdad entre sexos y salud, hasta los problemas derivados del cambio climático, recursos naturales y la pobreza, entre otros (UNESCO, 2012).

Ha habido multiplicidad de propuestas para abordar este reto, las cuales incluyen el desarrollo de una educación para la ciudadanía planetaria, que recoge las ideas inclusivas del paradigma educativo postmoderno, una educación para la democracia y participación social, donde se abordarían aspectos más prácticos, como los tecnológicos y de la producción, o educar para la paz en un mundo multicultural, demandando en este caso acciones éticas en una visión ecocéntrica (Novo \& Zaragoza, 2009).

Todas estas propuestas han chocado directamente contra las directrices educativas impuestas a las escuelas. Estas son vistas como instalaciones productivas, cuya misión principal es proveer a la industria de capital humano, y los docentes deben de rendir cuentas de ello ante las administraciones responsables (Hyslop-Margison \& Sears, 2007). La última reforma universitaria aplicada en España ha significado, de hecho, un paso adelante en este sentido. Se considera a la universidad, el último eslabón de la cadena educativa, como un preparatorio para la entrada del capital humano a la industria, sumando de esta forma enteros al PIB nacional. Esta mentalidad, forjada durante la revolución industrial, ha moldeado nuestra visión del trabajo, el capital, y, por tanto, la docencia, alrededor de los conceptos del derecho natural a la propiedad, de Locke, el beneficio social de perseguir el interés económico personal, de Adam Smith, la competitividad como valor inherente a la naturaleza humana, de Hobbes, y la tendencia general de marchar hacia el progreso, de Darwin (Clark, 1989). Todo esto, unido a la noción de que podemos acceder a la verdad por la observación y experimentación, tan asociada a la visión mecanicista racionalista de Newton y Descartes. Así, se ha desarrollado una dicotomía hombre/naturaleza implícita en el pensamiento occidental y en el subconsciente de todos nosotros.

\section{Hacia un nuevo marco educativo}

Ante el contexto en el que nos encontramos, cabría preguntarse: ¿cuál sería la mejor estrategia para promover la educación de ciudadanos con un pensamiento crítico que aportaran nuevos valores a una sociedad sostenible? El mencionado rechazo al cambio de paradigma educativo es debido a la pervivencia 
de los valores y creencias de la sociedad en general, surgidas, como hemos visto, durante la revolución industrial y el nacimiento de la economía de mercado occidental. Un cambio de paradigma educativo implicaría la reforma de todos esos valores imbuidos inconscientemente en la mente de todos nosotros, y eso, aunque deseable, no es probable que tenga lugar sin un detonante como el colapso de nuestra sociedad, bien por motivos sociales, económicos o medioambientales ¿hay, pues, alternativa a esta aparente paradoja inmovilista frente a la necesidad imperiosa de cambio social y medioambiental? A continuación, se proponen una serie de actuaciones educativas precisamente para abordar esta cuestión. Como base de estas actuaciones educativas se encuentra la convicción de que para acometer cualquier cambio no vale con mostrar el camino, lo cual se ha demostrado a todas luces insuficiente, sino que son los propios individuos los que deben encontrarlo y hacerlo suyo. La educación podrá facilitar la emergencia de estos individuos, con las actitudes, aptitudes y valores necesarios.

\subsection{Axiomas educativos para una enseñanza en la sostenibilidad}

Un paradigma es una forma de ver el mundo, de interpretarlo en un marco de referencia. El comportamiento consumista y derrochador de nuestra sociedad no parece que se desprenda de ninguno de los paradigmas educativos revisados anteriormente, los cuales son supuestamente la base de las leyes educativas actuales, la mayoría de las cuales persiguen la formación de personas íntegras y críticas ¿Qué explicación tiene esta aparente contradicción? Una explicación plausible podría deducirse a partir de la idea de que lo importante a la hora de formar ciudadanos críticos no son los supuestos teóricos subyacentes en las leyes educativas sino las metodologías didácticas llevadas a cabo en su desarrollo y las vivencias que son experimentadas durante el aprendizaje. Parte del problema puede estar, pues, en que los docentes no son conscientes del paradigma docente subyacente en su quehacer diario, sino que trabajan gobernados por los paradigmas sociales vigentes. F. Capra, (Capra, 1986) propuso una ampliación de paradigma al ámbito social, definiéndolo como "una constelación de conceptos, valores, percepciones y prácticas compartidas por una comunidad que forma una visión particular de la realidad que fundamenta el modo en el que la comunidad misma se organiza". Esta disyuntiva, paradigma educativo frente al paradigma social dominante, sería pues la que está detrás de la paradoja inmovilista. En el ámbito científico, los paradigmas no coexisten, sino que son suplantados por otros cuando existe una acumulación suficiente de evidencias que lo sustentan (Khun, 2012). El cambio de paradigma no se impone, sino que se sustancia a través de la lógica científica en la mente de la comunidad de investigadores. Este proceso sufre con frecuencia gran rechazo inicial pues el arraigo de los marcos de pensamiento es fuerte en las personas. Cuando hablamos de paradigmas sociales es posible que nos aproximemos más a la idea de los arquetipos e inconsciente colectivo de C. Jung (Jung, 2009) y, como hemos comentado anteriormente, son difícilmente cambiantes. Las acciones puntuales docentes, seminarios, clases, etc., o los intentos de cambio de paradigma educativo difícilmente pueden traspasar el paradigma social 
consumista en una sociedad tan dócil como la actual. Antes que actuar sobre el paradigma educativo habría que proporcionar a los docentes herramientas que les permitan llevar a cabo acciones y vivencias compatibles con esa visión crítica que pretendemos desarrollar en los individuos para iniciar el cambio hacia la sostenibilidad.

En ciencia se llaman axiomas a las proposiciones iniciales que se consideran evidentes y que permiten construir el conocimiento bajo esas premisas. Usando la misma analogía, y ya que el propósito que nos hemos marcado en estos momentos es diseñar acciones y vivencias que nos permita construir o formar personas, es tentador definir en este marco, el docente, una serie de axiomas o postulados que usen los docentes para construir tales acciones y vivencias necesarias para una educación transformadora. Un axioma docente debe ser visto como una herramienta de diseño, y sería compatible con los objetivos y contenidos en los planes de estudio actuales. La estrategia es cambiar la forma de pensar de las personas utilizando herramientas pedagógicas que nos permitan diseñar vivencias, las cuales educarían a individuos capaces de observar la verdadera esencia de los paradigmas docentes originales.

Una sociedad se podría, pues, cambiar desde dentro sin modificar el sistema educativo. La aplicación sistemática de una serie de axiomas por parte de los docentes trabajaría como despertador de conciencias, aflorando la posibilidad de cambio y la no inmutabilidad del statu quo social, y todo bajo una ética transformadora. El alumno debe de conocer y manipular las leyes de Newton en física, pero también debe de ser consciente de que las leyes son paradigmas bajo los cuales se interpreta la realidad y que como tales son cambiantes, y no inamovibles. Un alumno debe de conocer historia de España, pero a la vez darse cuenta de que la Historia con mayúsculas está hecha por pequeñas historias, en minúsculas, personales, donde los deseos, ambiciones, amores, casualidades y en definitiva, un contexto complejo, interdependiente y personal está detrás de ella. Un alumno de Derecho debe conocer las leyes, pero, a la vez, debe de saber que, tal como decía Tácito, cuanto más corrupto es un Estado, más leyes tiene.

Los axiomas educativos que se propondrán a continuación deben ser vistos con esa intención transformadora, con la esperanza de que a medio plazo su aplicación nos lleve a una sociedad más sostenible.

\subsubsection{La toma de decisiones en el uso de materia y energía debe estar basada en la pirámide del consumo/desechos}

Si hay algo evidente en la comunidad científica es que para lograr una sociedad sostenible es necesario dejar de lado la idea del crecimiento económico como motor del progreso (Victor, 2010). Este cambio de comportamiento llevaría aparejado una reducción del consumo de materia y energía. Esta idea, aunque evidente a la lógica, no ha calado en la conciencia de la sociedad. El tecno optimismo rei- 
nante nos hace pensar que surgirán soluciones a cada uno de los problemas con los que nos enfrentamos, desde puramente tecnológicos a los medioambientales, sin tener en cuenta que las actuaciones sobre sistemas complejos son de consecuencias inherentemente impredecibles. En realidad, es más conveniente buscar soluciones simples de menos calado tecnológico, pero más eficientes (Hueseman \& Hueseman, 2011). La progresiva implantación de energías renovables es un ejemplo de este tecno optimismo, sin embargo, las energías renovables no podrán suplantar totalmente el consumo de energía de origen fósil que demanda la sociedad en cantidades crecientes (Zehner, 2012). Actualmente se están desarrollando las bases de una ingeniería verde que, entre sus principios, reconoce el deber de buscar la eficiencia en el uso de la materia y energía, reduciendo su uso todo lo posible (Mulvihill et al., 2011).

Todas estas evidencias permiten proponer como primer axioma para la docencia a la pirámide del consumo, o de los desechos, como guía para un uso responsable de los recursos naturales (Figura 1) (Byrne \& Fitzpatrick, 2009). En su diseño original se concibió como la pirámide de los desechos, sin embargo, aquí se propone su uso ambivalente como pirámide del consumo/desechos, ya que ambos comportamientos pueden seguir pautas similares, pues tanto la reducción de la generación de materiales de desecho, principalmente agentes tóxicos contaminantes, como la reducción del consumo de materia y energía, son igualmente importante para lograr la sostenibilidad.

De un buen empleo de este axioma a nivel educativo surgiría una jerarquía de prioridades que debería ser puesta en práctica en todas las decisiones cotidianas en el aula que impliquen el uso de energía y materiales, y emergería de forma natural el diseño racional como estrategia para implementar este axioma. Se podría aplicar, por ejemplo, en pensar soluciones y diseños a problemas como el sistema de iluminación, diseño del aula, alimentación, vestido o uso de materiales electrónicos y papelería. La finalidad es que esta pirámide estuviera presente en los alumnos en su toma de decisiones diarias en todos los ámbitos de su vida.

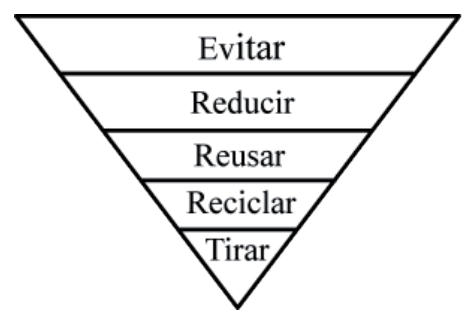

Figura 1. Pirámide del desecho/consumo. Fuente: (Byrne \& Fitzpatrick, 2009) 


\subsubsection{El juego es la vivencia primordial en el aprendizaje}

El mercantilismo ha ido progresivamente ocupando un lugar prioritario en la vida de las personas, especialmente la de los niños, hasta ocupar un lugar central, y su consecuencia más grave es la creciente pérdida del tiempo de juego de los niños (Linn, 2010). Las Naciones Unidas incluyeron el derecho al juego en su convención sobre Los Derechos del Niño de 1989, reconociendo el papel central del juego en el desarrollo saludable de los niños. De hecho, el juego creativo es fundamental para desarrollar la curiosidad, el razonamiento, la empatía, el compartir, la cooperación y un sentido sano de la competencia, emergiendo capacidades como la resolución constructiva de los problemas, el pensamiento divergente y la capacidad de autocontrol (Isenberg \& Quisenberry, 2002). A pesar de que estos beneficios del juego son ampliamente reconocidos, el tiempo que niños de entre 6 y 8 años dedican a este, ha disminuido en un tercio, parejo a la disminución de la importancia que los padres y educadores dan al juego (Linn, 2010). Así mismo, se ha constatado una reducción paulatina del tiempo que se dedica al juego libre y actividades artísticas y musicales en Estados Unidos (Bassok, Latham \& Rorem, 2016). El papel central que debe de tener el juego en la educación debe de traducirse no solo en aumentar el tiempo de juego, sino también en una disminución del uso de juguetes y otros aparatos tecnológicos que reducen la creatividad y el juego en grupo. Los beneficios del juego sobre el desarrollo del niño son evidentes, y su influencia sobre una concienciación de pertenencia a la naturaleza se puede conseguir induciendo el juego en espacios naturales, sin ninguna otra herramienta que lo que el niño encuentra en su entorno natural (Hattie, Marsh, Neill \& Richards, 1997).

\subsubsection{La tecnología por y para el servicio a las personas}

La tecnología ha jugado un papel esencial en la gran explosión del desarrollo industrial. Su uso se ha popularizado de tal forma que no concebimos ya nuestra vida en un entorno no tecnológico. De hecho, los medios tecnológicos dictan los fines sociales; la tecnología ya no es vista como un recurso para solucionar un problema previo, sino como un fin en si misma y el problema es cómo llevar la tecnología al mayor número de personas posible (Hueseman \& Hueseman, 2011). Esta reversión de los fines y los medios es, a juicio de Paul y Anne Ehrlich (Ehrlich \& Ehrlich, 2004), contraproducente, ya que la tecnología con frecuencia se propone "hacer de una forma muy eficiente aquello que nunca se debió de hacer". En cierto sentido la tecnología ha significado la pérdida de conexión del ser humano con el entorno natural. Se ha demostrado que el uso elevado de tecnologías de pantalla en población juvenil, como la televisión, videojuegos o los teléfonos móviles, se puede tomar como índice predictivo de una mala salud (Rosen et al., 2014). La tecnología también ha irrumpido en la educación, y su uso ha dado entrada al mundo de la información a través de internet. Los educadores deben de esforzarse por educar en el uso de la tecnología, desde la búsqueda de información fiable y relevante hasta trasladar una visión 
de la tecnología más apropiada para una sociedad sostenible, de manera que se conciba una tecnología local, descentralizada, a pequeña escala y simple, y que permita un trabajo lleno de sentido y satisfacción (Hueseman \& Hueseman, 2011).

\subsubsection{El objetivo de la relación entre hombres y mujeres es la cooperación, no la dominancia o competición.}

Ha habido una tendencia en la sociedad a ver en el hombre una inclinación natural, genéticamente codificada, al sexo y la promiscuidad, mientras que en la mujer esa inclinación natural se dirige hacia la elección de un hombre que haga de padre/cuidador. Esta disparidad de comportamientos ha tenido incluso cierto apoyo científico basado en interpretaciones hiperdarwinistas de la selección natural y la competición por los recursos. En realidad, no está demostrado que estos dos estereotipos tengan una justificación genética o evolutiva. Una explicación más plausible es que el ser humano se adapta a vivir bajo distintos comportamientos en diferentes contextos, siendo el estrés, originado por ejemplo por una escasez de recursos, aquella situación que genera en el hombre una situación de agresividad que desemboca en un comportamiento de dominancia física y psíquica sobre la mujer (Sanday, 1981). La mayor capacidad de adaptación y empatía de las mujeres con frecuencia lleva a la adopción de actitudes dóciles respecto a la dominancia del hombre. Este comportamiento tiene especial relevancia en la infancia y juventud, donde la educación refleja estos comportamientos sociales arquetípicos y se preservan en la sociedad (Chisamya, DeJaeghere, Kendall \& Khan, 2012). Un sistema educativo que persiga comportamientos sostenibles debe de incidir activamente en políticas inclusivas de la mujer. De hecho, una correcta educación de género ha sido descrito como uno de los indicadores más rápidos y fiables de una sana política democrática en un determinado país (Balaev, 2014). La cooperación entre géneros puede darse a múltiples niveles, y es favorecido por una tendencia natural a la cooperación, de tal manera que durante el desarrollo de juegos cooperativos se ha encontrado que el género es una variable neutra, de manera que la cooperación entre ambos sexos tiene lugar de forma espontánea (Lavega, Planas \& Ruiz, 2014). En este ámbito, habría que dedicar una especial atención al lenguaje, ya que determinados productos culturales, como el lenguaje y su narrativa, median el pensamiento y dejan su impronta en nuestra representación de la realidad (Bruner, 1991). Así, un lenguaje sexista puede impedir a las propias mujeres ser conscientes de su propia idiosincrasia, y reputados lingüistas abogan por una clara distinción entre género y sexo en el lenguaje hablado y escrito (Meseguer, 1991).

\subsection{Una epistemología para el aprendizaje y razonamiento}

Un mundo sostenible no sería un mundo simple, aunque pretendamos reducir la escala de nuestra economía, por lo que las personas que sostengan ese mundo deben de tener la capacidad de interpretar, 
vivir e interconectar con un entorno complejo. Se hace necesario, pues, facilitar una educación que provea de la epistemología necesarias para vivir en estos entornos y, aún más importante, que induzcan los cambios necesarios partiendo de la sociedad actual. Recientemente, K. Bain (Bain, 2012) condujo un estudio destinado a conocer cómo piensan y actúan aquellas personas más exitosas de la sociedad, en cuanto a su creatividad, iniciativa y capacidad transformadora. Este autor concluyó que estos individuos llevaban a cabo un aprendizaje profundo (deep learning) frente al aprendizaje superficial (surface learning) que caracteriza a otros estudiantes menos exitosos. El aprendizaje profundo conlleva un estado de conocimiento profundo que, a su vez, influye en los juicios y valoraciones sobre decisiones difíciles en la vida, permite obtener una retroalimentación sobre el pensamiento, hacer cuestiones, recoger evidencias y extraer conclusiones. Una de las premisas para alcanzar este tipo de aprendizaje es que durante el estudio se establezcan múltiples conexiones significativas con lo aprendido previamente y que el individuo tenga una curiosidad motivadora. No todos los individuos llegan a alcanzar un aprendizaje profundo, ya que el tipo de aprendizaje que tiene lugar está íntimamente ligado a las creencias epistemológicas que cada individuo tiene en un momento dado de su vida. Está ampliamente aceptado el modelo desarrollista que explica la evolución del conocimiento y aprendizaje durante el desarrollo del individuo (Kuhn, 1999). Según este modelo, existen personas con una epistemología absolutista, en la cual lo conocido es cierto, sin problemas, verdadero o falso y no necesita justificación, pues proviene de la observación de la realidad o de alguna autoridad. Esta epistemología es característica, aunque no exclusiva, de la niñez. Por contra, la persona multiplicista ve el conocimiento como algo ambiguo e idiosincrático, y está convencido de que cada individuo posee sus propias creencias y verdades. Esta visión es considerada típica de la adolescencia. Finalmente, un individuo evaluativo cree que hay unos métodos comunes para interrogar y adquirir conocimiento, por tanto, ciertas posiciones son más aceptables y justificadas que otras, y se suele dar bien entrada la edad adulta. Este individuo tiene un conocimiento profundo de la naturaleza y un conocimiento justificado, el cual implica un proceso activo de reflexión sobre el mismo acto del razonamiento. Es un estado de equilibrio entre la visión absolutista, objetiva, y la visión multiplicista, subjetiva, del conocimiento (Mason \& Boscolo, 2004).

Reflexionando sobre estos cambios, nos damos cuenta que, a medida que crece, el individuo va aumentando progresivamente la complejidad de su pensamiento y conocimiento, acercándose más al mundo que trata de comprender, el cual es un sistema complejo. A modo de paradoja podemos afirmar que un sistema complejo, nuestro cerebro, trata de comprender otro sistema complejo, nuestro entorno. En este proceso el individuo es objeto y, a la vez, sujeto del conocimiento, ya que no puede sustraerse de aquello a conocer, y el mismo acto de observar implica una forma de interactuar con el sistema. Sólo individuos con una visión evaluativa del conocimiento y un entendimiento de los sistemas complejos podrán comprender nuestra sociedad actual, y actuar para cambiarla hacia un modelo sostenible. Podríamos decir que vivimos en una sociedad adolescente, y necesitamos avanzar a la madurez para adquirir esa masa crítica de ciudadanos maduros. 
Los sistemas complejos son conjuntos de objetos, individuos o nodos interconectados de tal manera que generan un patrón propio de comportamiento resiliente en el tiempo, y las respuestas del sistema a las fuerzas externas son complejas, no lineales e impredecibles (Meadows, 2008). Una epistemología que reconozca la complejidad como base y objeto del conocimiento facilitaría, pues, un aprendizaje profundo, y los profesionales docentes deben de ser conscientes de esto. El profesorado debería de tener una buena formación en sistemas complejos, y saber que, si queremos comprender estos sistemas, debemos de saber que las fronteras entre los sistemas, tanto físicos como conceptuales, son sólo establecidas por palabras, pensamientos, percepción o acuerdo social, o sea construcciones mentales artificiales. En realidad, es en esas fronteras donde existe una mayor complejidad y diversidad. Otro concepto importante del que deberían ser conscientes es de nuestra racionalidad limitada cuando tomamos decisiones en un entorno complejo. En este sentido, nuestra toma de decisiones va a estar condicionada por nuestro conocimiento del sistema y por las influencias y condicionantes que el propio sistema ejerce sobe nosotros, y diferentes actores probablemente tomen las mismas decisiones cuando son situados en las mismas condiciones; es por ello que para ejercer un cambio de decisiones es necesario que cambien también las limitaciones a las que está impuesto el sujeto, rediseñando también el sistema (Meadows, 2008). Finalmente, tal como recalca F. Capra (Capra \& Luisi, 2014), el pensamiento en sistemas, o sistémico, es contextual, por tanto opuesto al pensamiento analítico, el cual promueve el separar para entender. El pensamiento sistémico coloca al objeto de estudio en el contexto de un todo mayor para así lograr su comprensión.

\section{3 Ética para una sociedad sostenible}

Tradicionalmente, las religiones han atribuido el origen de la ética a la existencia de un Dios, mientras que el empiricismo dominante actual lo justifica como meras adaptaciones evolutivas con cierta imprimación genética. No obstante, no es necesario acudir a las raíces u origen de la ética para intuir que necesitamos imponernos unas ciertas limitaciones en nuestro comportamiento social. El propio razonamiento epistemológico evaluativo lleva implícito el advenimiento de cierta ética: no sólo existe lo bueno o lo malo, sino que también hay una amplia zona intermedia, y es precisamente en esta zona donde transcurre la mayor parte de nuestra vida en sociedad, en concordancia con lo que nos dice la teoría de sistemas complejos acerca de las fronteras. También intuimos, según esta epistemología, que debe de haber una ética común, en contraposición a la ética personal y única para cada individuo tan en boga actualmente. Siguiendo con nuestro razonamiento, una sociedad madura, en sentido epistemológico, evaluativa, implementaría en el sistema educativo la enseñanza de esa ética común como necesaria para lograr una sociedad justa.

Llegados a este punto, pareciendo necesario el consensuar una ética común, ¿qué clase de ética sería esa? ¿necesitaríamos una ética especial para lograr una sociedad sostenible? Todas estas cuestiones se 
están tratando de abordar desde las propias religiones, ya que una visión religiosa del mundo condiciona el comportamiento frente al medio ambiente, y si la actual crisis medioambiental puede ocasionar una crisis religiosa, desde los propios estamentos religiosos se requiere un reexamen de sus tradiciones para abarcar las ideas ecológicas necesarias para desarrollar concepciones del mundo más sostenibles. La sociedad global puede no necesitar buscar respuestas en las tradiciones religiosas, pero sí necesita algo parecido al servicio religioso para dar sentido a sus objetivos y complejidad (Jenkins \& Chapple, 2011). De estos intentos surgen nuevas cuestiones básicas: ¿qué debemos de sostener? ¿cómo se deben de relacionar los humanos y los sistemas ecológicos? ¿cuáles son las condiciones que sustentan el espíritu humano? En otro nivel, también se está avanzando en el establecimiento de una ética mundial promovida por las Naciones Unidas, acogiendo una visión humanitarista, enraizada en la regla de oro de la ética, o principio de reciprocidad, que apremia a los individuos a tratar al prójimo como quisiera ser tratado a sí mismo (Küng, 2011). Esta ética no inculca derechos, sino responsabilidades, una cultura de no violencia y respeto a toda forma de vida, solidaridad, tolerancia e igualdad y colaboración entre hombre y mujer.

La ética humanitarista es necesaria, pero no suficiente para una sociedad ecocéntrica, por eso es necesario el avance de la interacción entre religión y ecología: no es cuestión de hacer las cosas a la manera de la naturaleza, sino más bien decidir qué formas de la naturaleza queremos establecer, mantener, restaurar o cambiar (Jenkins \& Chapple, 2011).

Como se ha mencionado anteriormente, la educación no tiene por qué enseñar ética ni religión como asignatura, pero ni profesores ni alumnos pueden vivir de forma aséptica, éticamente hablando, durante el proceso de enseñanza (Clark,1989). Cada acción de este proceso lleva implícita cierta ética, y es esta ética implícita, vivida, la que el alumno aprende. Es más, se han propuesto actividades o vivencias que pueden incidir en la adquisición de un bienestar subjetivo, como la práctica de meditación mediante la técnica del mindfulness en colegios (Ericson, Kjønstad \& Barstad, 2013).

\section{Conclusiones finales}

La puesta en práctica de una educación para la sostenibilidad, como la propuesta en este trabajo, es algo que, en cierto sentido, debería formar parte del imperativo moral de cada uno de nosotros como educadores. Ya que no está en nuestras manos el cambio normativo y legislativo necesario, el marco educativo que se propone es compatible con las leyes educativas actuales. No obstante, para lograr una amplia diseminación de los conceptos propuestos, sería necesario actuar sobre el profesorado en formación, con el objetivo de dotarle de los axiomas educativos descritos previamente para que los usara como herramientas de trabajo en el diseño de experiencias y vivencias educativas. Así mismo, debería abarcar la realidad mediante una epistemología de los sistemas complejos, interpretando tales sistemas 
como la forma en la que se estructuran y funcionan los sistemas naturales. Finalmente, debería adoptar, en su labor educativa, una ética ecocéntrica y humanitarista de modo que permee de forma pasiva a los estudiantes. Ello posibilitaría el inicio de una nueva etapa educativa, donde personas libres, críticas y con valores éticos sólidos hicieran posible el establecimiento de una sociedad sostenible.

\section{Referencias}

Appleton, A. F. (2006). Sustainability: A practitioner's reflection. Technology in Society 28, 3-18. doi:10.1016/j.techsoc.2005.10.001

Bain, K. (2012). What the Best College Students Do. Belknap Press.

Balaev, M. (2014). Improving models of democracy: The example of lagged effects of economic development, education, and gender equality. Social Science Research 46(C), 169-183. doi:10.1016/j.ssresearch.2014.03.004

Barr, R. B., \& Tagg, J. (1995). Barr and Tagg, From Teaching to Learning. Change 27, 12-26. doi. org/10.1080/00091383.1995.10544672

Bassok, D., Latham, S. \& Rorem, A. (2016). Is Kindergarten the New First Grade?. AERA Open 1, $1-31$.

Bruner, J. (1991). The narrative construction of reality. Critical Inquiry 18, 1-21.

Burger, J. R., Allen, C. D., Brown, J. H., Burnside, W. R., Davidson, A. D., Fristoe, T. S... Zuo, W. (2012). The Macroecology of Sustainability. PLoS Biology 10, e1001345. doi:10.1371/ journal.pbio.1001345.s001

Byrne, E. P., \& Fitzpatrick, J. J. (2009). Chemical engineering in an unsustainable world: Obligations and opportunities. Education for Chemical Engineers 4, 51-67. doi:10.1016/j. ece.2009.09.001

Capra, F. (1986). The Concept of Paradigm and Paradigm Shift. Re-Vision 4, 51-67. doi:10.1016/j. ece.2009.09.001

Capra, F., \& Luisi, P. L. (2014). The systems view of life. Cambridge University Press.

Chisamya, G., DeJaeghere, J., Kendall, N., \& Khan, M. A. (2012). Gender and Education for All: Progress and problems in achieving gender equity. International Journal of Educational Development 32, 743-755. doi:10.1016/j.ijedudev.2011.10.004 
Clark, M.E. (1989). Ariadne's Thread: The Search for New Modes of Thinking. Palgrave Macmillan.

Daily, G. C., \& Ehrlich, P. R. (1996). Population, sustainability, and Earth's carrying capacity. BioScience 42, 761-771. doi: 10.2307/1311995

de Castro, C., Mediavilla, M., Miguel, L. J., \& Frechoso, F. (2013). Global solar electric potential: A review of their technical and sustainable limits. Renewable and Sustainable Energy Reviews 28, 824-835. doi:10.1016/j.rser.2013.08.040

Dean, D., Jr, \& Kuhn, D. (2007). Direct instruction vs. discovery: The long view. Science Education 91, 384-397. doi:10.1002/sce.20194

Dewey, J. (1910). How we think. D.C. Heat \& Company.

Dewey, J. (2013). My pedagogic creed. KKIEN Publ. Int.

Ehrlich, P. R., \& Ehrlich, A. (2004). One With Nineveh: Politics, Consumption, and the Human Future. Island Press.

Ericson, T., Kjønstad, B. G., \& Barstad, A. (2013). Mindfulness and sustainability. Ecological Economics. 104, 73-79. doi:10.1016/j.ecolecon.2014.04.007

Freire, P. (2005). Pedagogía del oprimido. Siglo XXI.

Georgescu, D., (2008). Philosophical "paradigms" of education: how philosophy impacts on learning. World Congress of Philosophy 2008 Proceeding 37, 40-55.

Harlem, B. G. (1987). Our common future. Rio De Janeiro: United Nations World Commission on Environment and Development (WCED).

Hattie, J., Marsh, H. W., Neill, J. T., \& Richards, G. E. (1997). Adventure education and outward bound: out-of-class experiences that make a lasting difference. Review of Educational Research 67, 43-87. doi: 10.2307/1170619

Hueseman, M., \& Hueseman, J. (2011). Tecno-Fix. New Society Publishers.

Hyslop-Margison, E. J., \& Sears, A. M. (2007). Neo-Liberalism, Globalization and Human Capital Learning. Springer.

Imran, S., Alam, K., \& Beaumont, N. (2011). Reinterpreting the Definition of Sustainable Development for a More Ecocentric Reorientation. Sustainable Development 22, 134-144. doi:10.1002/sd.537 
Isenberg, J. P., \& Quisenberry, N. (2002). A Position Paper of the Association for Childhood Education International PLAY: Essential for all Children. Childhood Education 79, 33-39. do i:10.1080/00094056.2002.10522763

Jenkins, W., \& Chapple, C. K. (2011). Religion and Environment. Annual Review of Environment and Resources 36, 441-463. doi:10.1146/annurev-environ-042610-103728

Jung, C. G. (2009). Arquetipos e insconciente colectivos. Paidos Ibérica.

Khun, T. S. (2012). The structure of scientific revolutions (50 ed). University of Chicago Pr.

Klahr, D., \& Nigam, M. (2004). The equivalence of learning paths in early science instruction: effect of direct instruction and discovery learning.. Psychological Science 15, 661-667. doi:10.1111/j.0956-7976.2004.00737.x

Kuhn, D. (1999). A Developmental Model of Critical Thinking. Educational Researcher 28, 16-46. doi:10.3102/0013189X028002016

Küng, H. (2011). Lo que yo creo. Editorial Trotta.

Lavega, P., Planas, A., \& Ruiz, P. (2014). Juegos cooperativos e inclusión en educación física. Revista internacional de medicina y ciencias de la actividad física y del deporte 14, 37-51.

Linn, S. (2010). Commercialism in children's lives. W. W. Norton \& Company.

Mason, L., \& Boscolo, P. (2004). Role of epistemological understanding and interest in interpreting a controversy and in topic-specific belief change. Contemporary Educational Psychology 29, 103-128. doi:10.1016/j.cedpsych.2004.01.001

Meadows, D. H. (2008). Thinking in systems. Chelsea Green Publishing Company.

Meseguer, A. G. (1991). Gender-sex clashes in Spanish: A semantic typology of animated nouns:(A contribution to the study of liguistic sexism). Journal of Pragmatics 15, 445-463. https:// doi.org/10.1016/0378-2166(91)90048-3

Mulvihill, M. J., Beach, E. S., Zimmerman, J. B., \& Anastas, P. T. (2011). Green Chemistry and Green Engineering: A Framework for Sustainable Technology Development. Annual Review of Environment and Resources 36, 271-293. doi:10.1146/annurev-environ-032009-095500

Novo, M., \& Zaragoza, F. M. (2009). El desarrollo sostenible: su dimensión ambiental y educativa. Editorial Universitas, S.A. 
Robinson, K. S. (2013). Is Sustainability Still Possible?. Island Press.

Rogers, D. S., Duraiappah, A. K., Antons, D. C., Munoz, P., Bai, X., Fragkias, M., \& Gutscher, H. (2012). A vision for human well-being: transition to social sustainability. Current Opinion in Environmental Sustainability 4 . doi:10.1016/j.cosust.2012.01.013

Rosen, L. D., Lim, A. F., Felt, J., Carrier, L. M., Cheever, N. A., Lara-Ruiz, J. M., Mendoza, J.S. and Rokkum J., (2014). Media and technology use predicts ill-being among children, preteens and teenagers independent of the negative health impacts of exercise and eating habits. Computers in Human Behavior 35, 364-375. doi:10.1016/j.chb.2014.01.036

Sanday, P. R. (1981). Female power and male dominance: on the origins of sexual inequelity. Cambridge University Press.

UNESCO. (2012). Forjar la educación del mañana: Decenio de las Naciones Unidas de la Educación para el Dessarollo Sostenible: informe 2012. UNESCO.

van Gorp, A. C. (2014). Ethical Issues in Engineering Design; Safety and Sustainability. Simon Stevin Series in the Philosophy of Technology , 1-224. uuid:39e544ba-15dc-4154-a6fce25106320e90

Victor, P. (2010). Questioning economic growth. Nature 468, 370-371. doi:10.1038/468370a

Wessendorf, S. (2010). The Multiculturalism Backlash: European Discourses, Policies and Practices. Routledge.

Zehner, O. (2012). Green Illusions. University of Nebraska Press.

WBCSD (2010). Business \& Development: Challenges and opportunities in a rapidly changing world. World Business Council for Sustainable Development. 